\title{
Application of the Hurst index to evaluate the testing of information gathering system components
}

\author{
Sergei Klevtsov ${ }^{1 *}$ \\ ${ }^{1}$ Institute of Radiotechnical Systems and Control, Southern Federal University, Taganrog, Russia
}

\begin{abstract}
The performance of the technical object is determined based on the evaluation of its parameters. Sensors of physical quantities are used to collect data on the values of the parameters of the controlled object. The performance evaluation of an object depends on the accuracy of the parameter measurement. The measurement accuracy is determined by the sensor conversion characteristic. If the sensor calibration tests are performed correctly, the conversion characteristic will accurately reflect the relationship between the measured parameter and the output electrical signal. A method for assessing the quality of the conversion characteristics of the microprocessor sensor, which is based on the use of Hurst index. The sensor of slowly changing physical quantities is considered. Based on the results of several test cycles obtained at a fixed ambient temperature, a series similar to the time series is formed. The initial series is subjected to additional processing before evaluation. The Hurst exponent is determined for the obtained series. The value of the Hurst index determines the quality of the test results. The possibility of using fractality index to assess the quality of tests is also considered.
\end{abstract}

\section{Introduction}

The performance assessment of a technical object is based on an analysis of a change in a parameter or a combination of its parameters $[1,2]$. The object is affected by climatic factors such as temperature, humidity, pressure and others, which lead to the degradation of the parameters of the object. Improper operation also affects the performance of the facility. An object fails when degradation exceeds the permissible level for a parameter change. This may cause an accident. Therefore, it is important to accurately measure the current value of the controlled parameter of the object $[3,4]$.

Parameter measurements are performed by microprocessor sensors of physical quantities. They are important components of a distributed microcomputer system for collecting information about the parameters of a technical object. The high accuracy of measurements made using a microprocessor sensor, largely depends on the quality of its conversion characteristics. This characteristic is formed on the basis of the test results for the calibration of the sensor. Tests are conducted according to a special program and test

*Corresponding author: sergkmps@mail.ru 
procedure. Any deviations from the methodology may lead to a decrease in the quality of the conversion characteristics. This deteriorates the metrological characteristics of the sensor. For example, the measurement error increases. The main danger is that these changes are difficult to capture.

\section{Formulation of the Problem}

The construction of the spatial characteristics of the transformation is important for microprocessor sensors, since along with the measured parameter, the influence of the main destabilizing factor is taken into account. Temperature is often such a factor. The conversion characteristic in this case is a function that defines the parameter depending on the values of the electrical signals coming from the temperature channel and the channel of the measured value [3].

Tests are carried out in cycles at a given ambient temperature. The parameter changes from the lower value to the upper value and vice versa. Several cycles of parameter change are performed. The temperature does not change. The procedure is performed for various fixed temperatures. Thus, the test results represent a periodically repeating dependence of the output electrical signal on the measured parameter at a fixed temperature [3]. The test procedure requires that the test cycles be independent. If the test procedure is violated, the results of the subsequent test cycle will be determined by the previous cycle. This will affect the dependency form of the subsequent cycle. It will differ from the dependency forms for previous test cycles.

Let there be results of tests of a pressure sensor in the form of $U=f(P, T)$, where $U$ is the output electrical signal, $P$ is the measured parameter (pressure), $T$ is the temperature. We highlight the test results obtained at a given temperature $T_{k}$. The $U_{k}$ array consists of several test cycles:

$$
U_{k}=\left\{U_{k 1}\left(P, T_{k}\right), \ldots, U_{k i}\left(P, T_{k}\right), \ldots, U_{k n}\left(P, T_{k}\right)\right\},
$$

where $U_{k i}\left(P, T_{k}\right)=\left\{u_{k i 1}, u_{k i 2}, \ldots, u_{k i j}, \ldots, u_{k i m}\right\}$ is the results of a separate test cycle; $i=1, n ; j=1, m ; u_{k i j}$ is the value of the output signal of the sensor at a pressure $P_{j}, n$ is the number of test cycles at a fixed temperature, $m$ is the number of values of the output electric signal obtained in the cycle. The values of $P$ in the cycle increase from the minimum $\left(P_{I}=P_{\min }\right)$ to the maximum value of $P_{\max }\left(P_{m / 2}=P_{\max }\right)$ and vice versa $\left(P_{m}=\right.$ $\left.P_{\max }\right)$.

To assess the quality of test results, it is necessary to determine whether or not the results of previous test cycles affect the results of subsequent cycles, how great is this effect, whether this effect can be neglected.

\section{The Use of the Hurst Exponent}

We construct a graph by including in it sequentially the results of all test cycles at a given temperature in the order of conduct. On the abscissa axis, we place the numbers of individual measurements in order of execution. Test cycles on the graph will be represented by elements similar in shape $[3,5]$. Ideally, these elements are equivalent to each other. They are actually a repetition of one element. But in a real situation, the elements are different. However, when performing the test procedure, these differences are insignificant and are taken into account in the measurement error. To confirm the quality of the tests, it is necessary to compare these elements with each other and evaluate how the elements of the graph coincide in shape. 
The test data presented in the above method can be considered similar to a time series. The pressure change step is constant throughout all test cycles. Therefore, for such an assessment, it is proposed to use the method of normalized range, based on the calculation of the Hurst exponent [5 - 10].

The Hurst exponent $\mathrm{H}$ is determined from the relation $[5,7,8,11]$ :

$$
R / S=(\tau / \alpha)^{H}
$$

where $R / S$ is the normalized range, $H$ is the Hurst exponent, $\alpha$ is the constant, usually taken equal to $0.5[8,9,11], \tau$ is the current value of the sample length.

The calculation scheme for $H$ is given in [7, 8, 11].

For this task, it is important to fix that $H<0.5$. In this case, the series is antipersistent and has pronounced fractal properties. This means the repeatability of the form and the correctness of the test [5]. If $H>0.5$, then the analyzed time series is persistent and has trend resistance. In this case, there is a significant difference in the forms of the various test cycles. Possible violation of the independence of the results of individual cycles of the experiment.

In fig. Figure 1 shows the temperature dependence of the Hurst exponent for three types of pressure sensors.

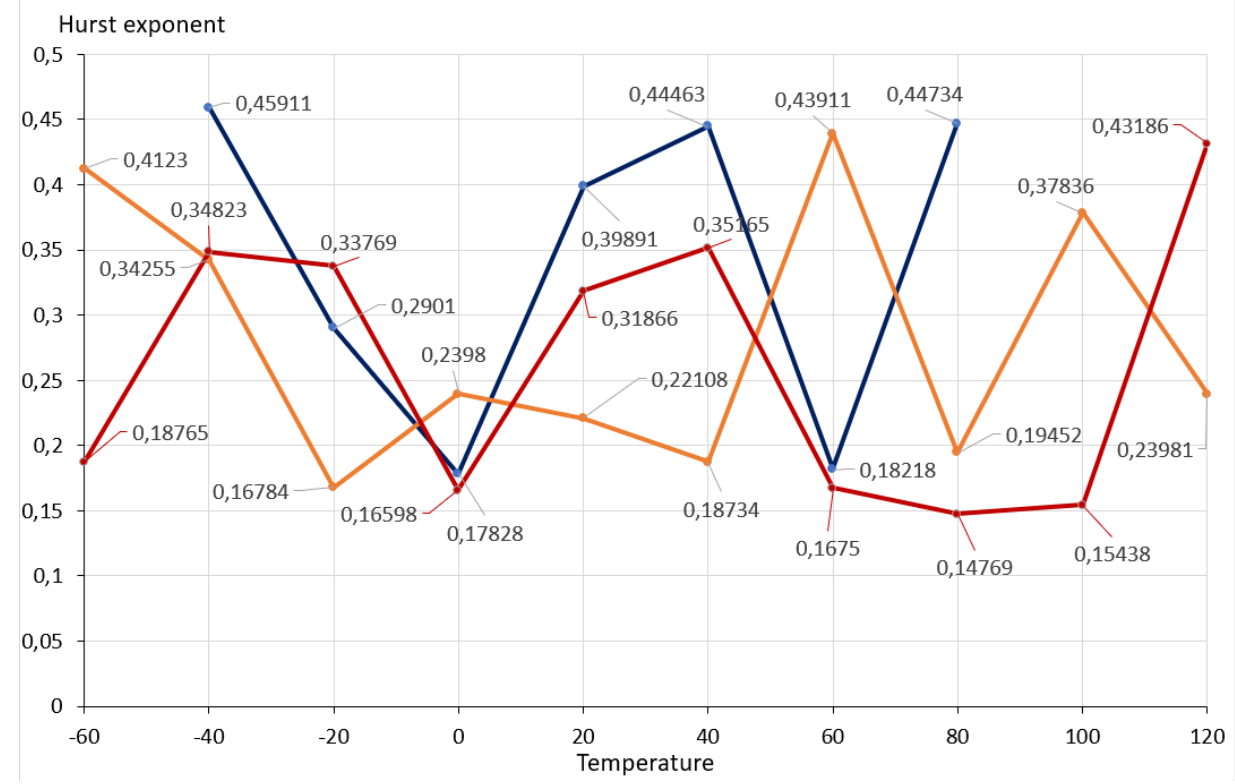

Fig. 1. Hurst exponent versus temperature.

Hurst values are less than 0.5 for all samples obtained from the test results. Each of the rows is antipersistent. The calculation results show that the series is made up of elements with a repeating shape. No significant dependence of individual test cycles on previous cycles has been identified. The calculation results show that the use of the Hurst exponent to assess the fractality of the test schedule is possible. However, it is necessary to determine how sensitive the method is.

Sensor tests were carried out in full accordance with the standards and test procedure. The first absolute pressure sensor is a strain gauge type for measuring pressure up to 0.6 $\mathrm{MPa}$ [5]. The other two sensors are also absolute pressure sensors with measuring ranges up to $5.880 \mathrm{MPa}$ and $0.980 \mathrm{MPa}$, respectively. The indicator is calculated using the formula 
(2). For the sensors under consideration, the number of points was determined in accordance with the scheme proposed in [5]. The number of points in the sample should be at least 7000 (Fig. 2).

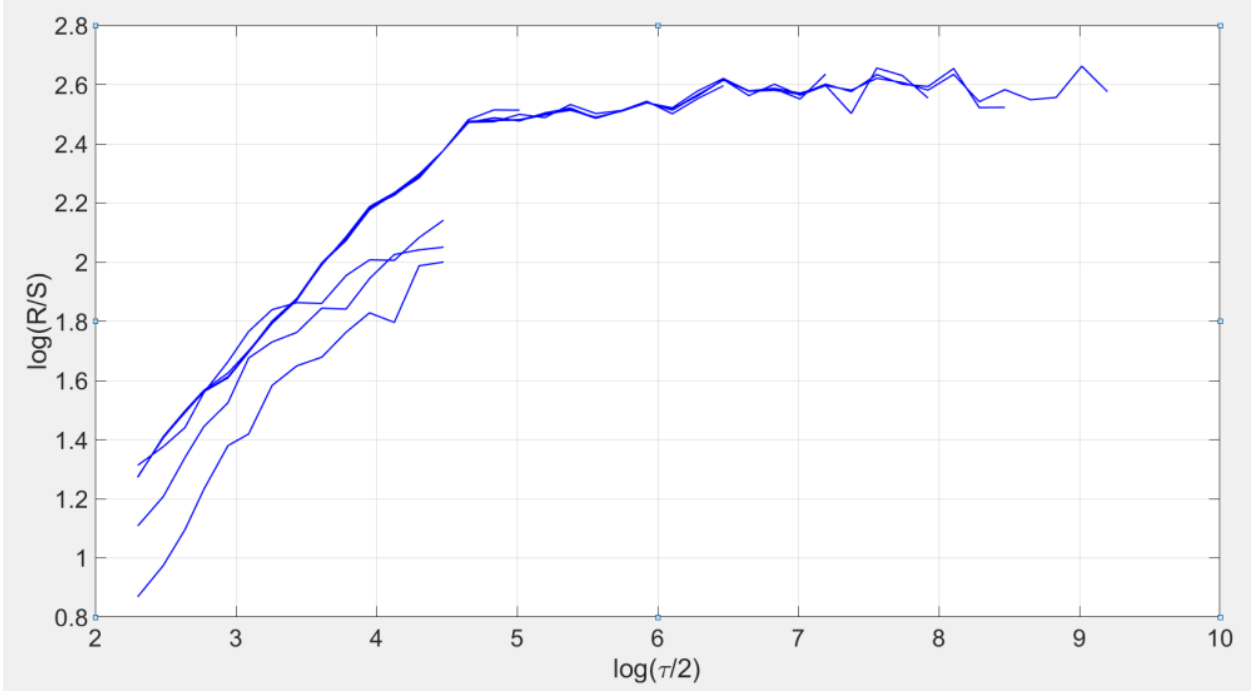

Fig. 2. Hurst exponent for different sample sizes.

Consider a violation of the test conditions associated with a decrease in the duration of the installation of a new temperature. In this case, the temperature conditions of previous tests will affect the results of current tests. In this case, the temperature of the sensor at the beginning of the first test cycle will not be equal to the required temperature. The output values will be distorted by the temperature of previous tests.

We will construct artificial series for individual reference test cycles of the sensor for measuring pressure up to 0.6 MPa. In fig. 3 shows the Hurst exponent for several consecutive test cycles. The temperature is $40^{\circ} \mathrm{C}$.

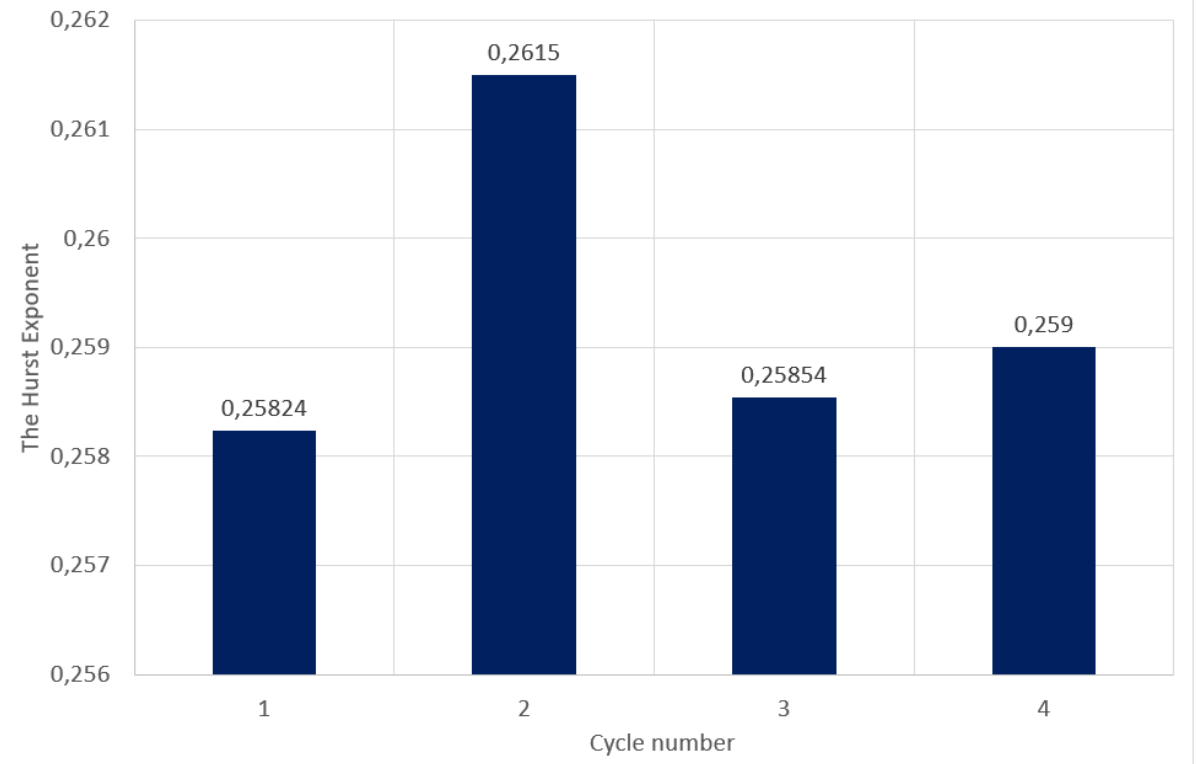

Fig. 3. Hurst exponent for individual test cycles. 
You can determine the range of acceptable values of $H$ for $\mathrm{T}=40^{\circ} \mathrm{C}$ :

$$
0,255<H<0,264 \text {. }
$$

To assess the sensitivity of the Hurst indicator to changes in the shape of the test cycle, reproducibility can be used. This metrological characteristic determines the difference between the output signals of the sensor in two test cycles:

$$
\delta_{r}=\frac{\Delta_{m}}{F S} * 100 \%
$$

where $\Delta_{m}$ is the maximum difference between the output values of the signal from the sensor obtained in two test cycles, FS is the range of the sensor output signal.

The results presented in fig.3, obtained with a reproducibility value of $0.0212 \%$. The absolute measurement error of the output signal was $0.02 \mathrm{mV}$.

If the temperature of the previous cycle affects the sensor output, the value $\Delta_{m}$ increases. This increase is noticeable in the initial part of the cycle. Computer simulation shows that the Hurst exponent does not meet the constraints (3) with an increase in signal spread in the initial part of cycle $\Delta_{m} \geq 0,3$. The results are shown in fig. 4 .

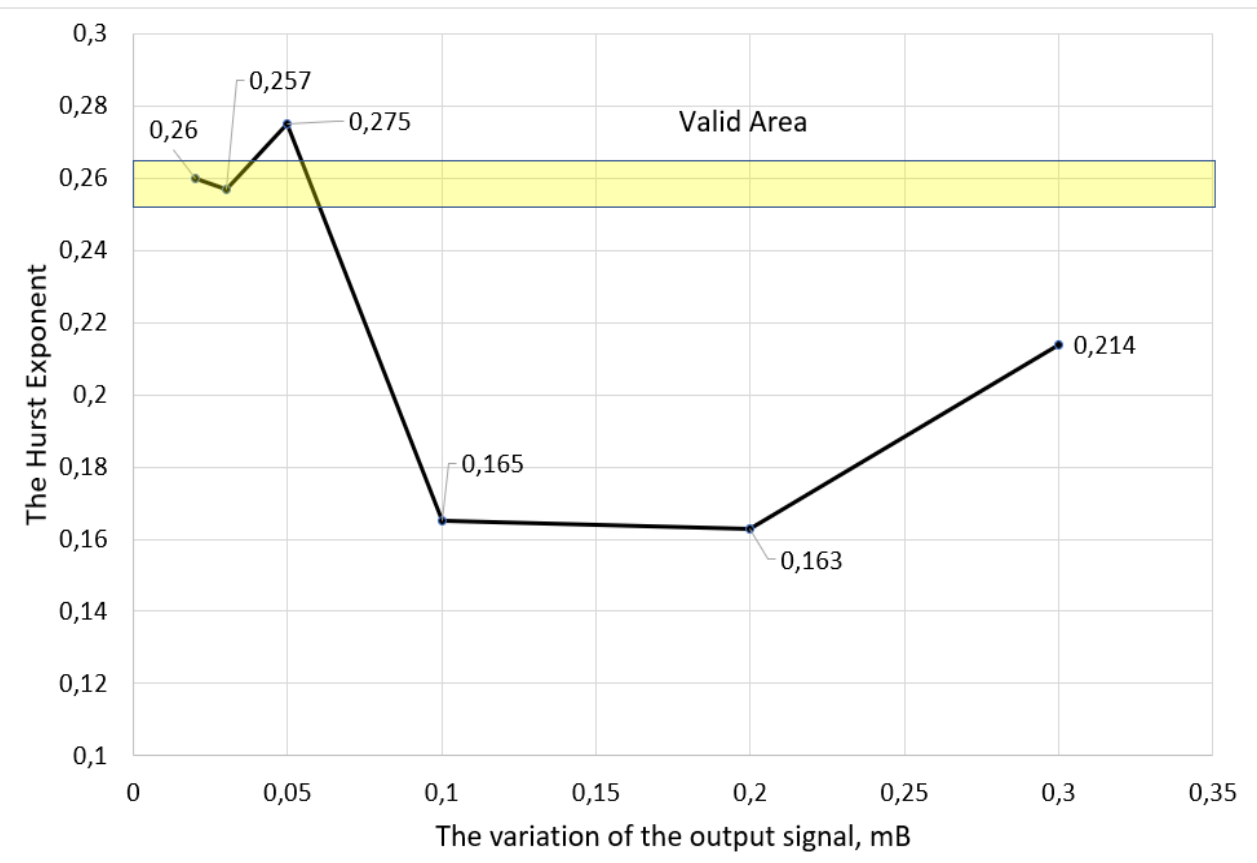

Fig. 4. Change in the Hurst exponent from the scatter of the output signal at $\mathrm{T}=40^{\circ} \mathrm{C}$.

With large values of $\Delta_{m}$, the Hurst exponent can be more than 0.5 , which characterizes serious violations of the test conditions or the sensor failure (fig. 5).

If we consider the series built on the basis of the results of two or more test cycles, then the value of the Hurst index increases, but does not exceed 0.5. This is natural, since the cycle data is different. In this case, the sensitivity of such series to the influence of temperature on the output signal at the initial stage of the first cycle decreases. 


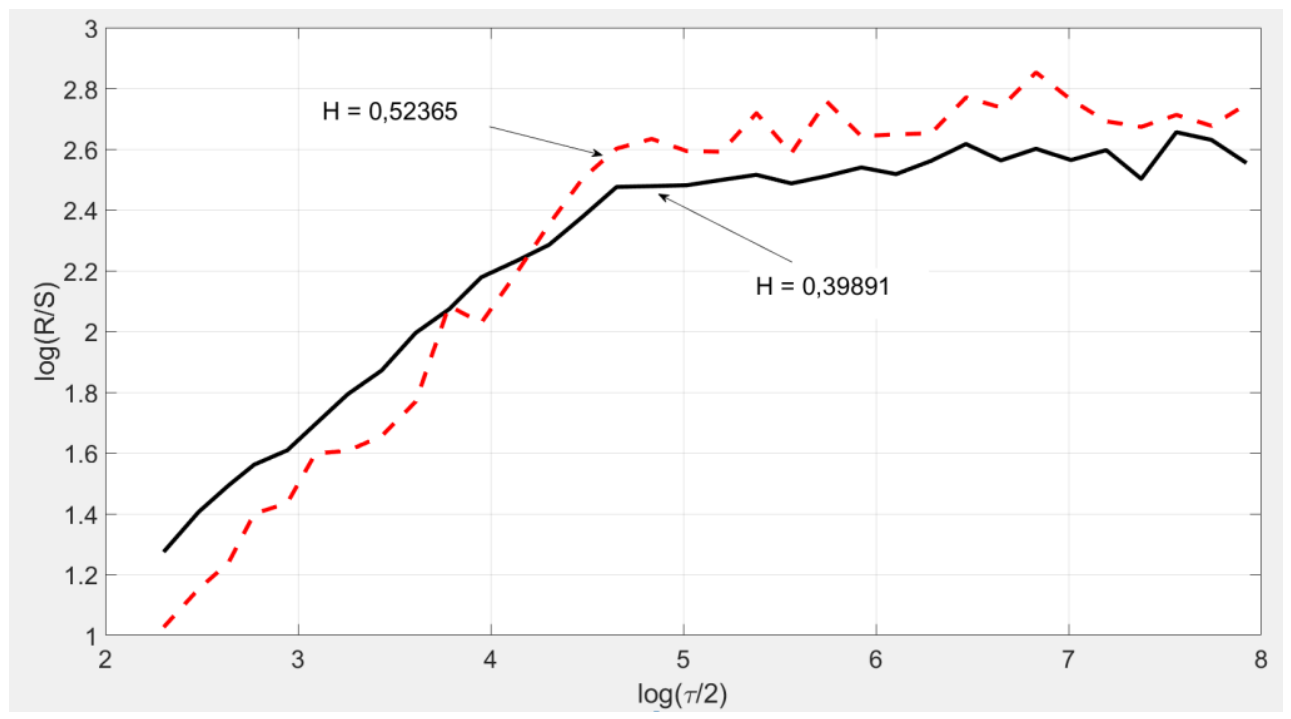

Fig. 5. Hurst exponent for the series of test results obtained at a temperature of $\mathrm{T}=20^{\circ} \mathrm{C}$.

\section{Conclusion}

The use of the Hurst indicator is advisable in assessing the quality of testing sensors. The conclusion is made on the basis of processing the results of tests of pressure sensors by the method of normalized magnitude. The Hurst index for a series built on the basis of the results of several test cycles at a fixed ambient temperature should be in the range of $(0.0 \div 0.5)$.

During testing, essential conditions for their performance may be violated. For example, the conditions for the transition to testing from one temperature to another may be violated. In this case, the temperature conditions of the previous cycle will affect the results of the current test cycle. To identify such violations, it is necessary to compare the Hurst indicator of the alleged poor test cycle with a range of acceptable values. This range is formed on the basis of processing the results of reference test cycles. If the Hurst indicator does not meet the restrictions, then the conditions of the previous test cycle affected the results of the analyzed cycle. If its value is more than 0.5 , then this signals a significant effect or a sensor failure.

\section{References}

1. S. Klevtsov, Identification of the State of Technical Objects Based on Analyzing a Limited Set of Parameters, 2016 International Siberian Conference on Control and Communications, SIBCON, 2016, Proceedings, Pp. 749-752 (2016)

2. Lihua Sun, Yingjun Guo, Haichao Ran, A New Method of Early Real-Time Fault Diagnosis for Technical Process, Electrical and Control Engineering (ICECE), 2010 International Conference, Wuhan, China, Pp. 4912 - 4915 (2010)

3. S. Klevtsov, Y. Udod, Model of the Spatial Conversion Characteristics for Graduation of the Microprocessor-Based Sensor's with Indemnification of Influence Destabilizing Factors, 2015 International Siberian Conference on Control and Comunications (SIBCON), Proceedings, Russia, Omsk (2015).

http://ieeexplore.ieee.org/stamp/stamp.jsp?arnumber=7,147,097. 
4. C. Bluemm, R. Weiss, R. Weigel, D. Brenk, Correcting nonlinearity and temperature influence of sensors through B-spline modeling, 2010 IEEE International Symposium on Industrial Electronics, Proceedings, Bari, Italy, Pp. 3356 - 3361 (2010) https://ieeexplore.ieee.org/document/5637991.

5. S. Klevtsov, Using the Method of Normalized Amplitude for Assessing the Quality of the Calibration Tests of the Pressure Sensor, 2019 Ural Symposium on Biomedical Engineering, Radioelectronics and Information Technology (USBEREIT), Yekaterinburg, Russia, Pp. 197-199 (2019)

6. J. Matuszewski, Application of clustering methods for recognition of technical objects, Modern Problems of Radio Engineering, Telecommunications and Computer Science (TCSET), 2010 International Conference, Pp. 39- 40 (2010)

7. H.E. Hurst, Long-term storage of reservoirs: an experimental study, Transactions of the American Society of Civil Engineers, Vol. 116, Pp. 770-799 1(951)

8. F. Roel Ceballos, Fe F. Largo, On the Estimation of the Hurst Exponent Using Adjusted Rescaled Range Analysis, Detrended Fluctuation Analysis and Variance Time Plot: A Case of Exponential Distribution, Imperial Journal of Interdisciplinary Research (IJIR), Vol. 3, Issue 8, Pp. 424-434 (2017)

9. Jerzy Wawszczak, Methods for estimating the Hurst exponent. The analysis of its value for fracture surface research, Materials Science-Poland, Vol. 23, No. 2, Pp.585-591 (2005)

10. N. P. Cousins, V. M. Loginov, The use of the normalized scope method in the analysis of speech pathologies of neurological genesis, COMPUTER RESEARCH AND MODELING, Vol. 6, No.5, Pp. 775-791 (2014)

11. F. Cervantes-De la Torre, J.I. Gonz'alez-Trejo, C.A. Real-Ramirez, L.F. Hoyos-Reyes, Fractal dimension algorithms and their application to time series associated with natural phenomena, Journal of Physics: Conference Series, No. 475, Pp.1-10 (2013) 